\title{
Thermodynamic Relation between Voltage-Concentration Dependence and Salt Adsorption in Electrochemical Cells
}

\author{
R. A. Rica, R. Ziano, D. Salerno, F. Mantegazza, and D. Brogioli \\ Dipartimento di Medicina Sperimentale, Università degli Studi di Milano-Bicocca, Via Cadore 48, Monza (MB) 20900, Italy \\ (Received 11 May 2012; revised manuscript received 6 July 2012; published 11 October 2012)
}

\begin{abstract}
Electrochemical cells containing two electrodes dipped in an ionic solution are widely used as charge accumulators, either with polarizable (supercapacitor) or nonpolarizable (battery) electrodes. Recent applications include desalination ("capacitive deionization") and energy extraction from salinity differences ("capacitive mixing"). In this Letter, we analyze a general relation between the variation of the electric potential as a function of the concentration and the salt adsorption. This relation comes from the evaluation of the electrical and mechanical energy exchange along a reversible cycle, which involves salt adsorption and release by the electrodes. The obtained relation thus describes a connection between capacitive deionization and capacitive mixing. We check this relation with experimental data already reported in the literature, and moreover by some classical physical models for electrodes, including polarizable and nonpolarizable electrodes. The generality of the relation makes it very useful in the study of the properties of the electric double layer.
\end{abstract}

DOI: 10.1103/PhysRevLett.109.156103

The electrochemistry of salt solutions and the related accumulator technologies recently found two potential applications with high social impact, namely, the energy production from the salinity difference between sea and river water, and sea water desalination. By "accumulator technologies" we refer to any mechanism where charge is temporarily stored in the electrodes of an electrochemical cell, either capacitively, in redox reactions, or through ion intercalation.

Traditional desalination techniques [1,2] allow production of water with lower salt concentration from a given solution, by consuming energy; they include reverse osmosis, electrodialysis, and distillation. A by-product of the process is a brine, i.e., a solution with higher salt concentration. Salinity-difference energy [2-4] can be seen as the reverse of desalination processes; i.e., it consists in the controlled mixing of two solutions with different concentrations, so that the Gibbs energy of mixing is extracted. The reverse of the above-cited desalination techniques are, respectively, pressure-retarded osmosis [5,6], reverse electrodialysis [7,8], and the vapor pressure method [9].

One of the above-mentioned accumulator technologies for desalination is the so-called "capacitive deionization" (CDI) [10]. Two activated carbon electrodes are dipped into the solution to be deionized, and are electrically charged, just as in a supercapacitor. During the charging phase, the electrodes adsorb a net amount of salt from the solution, thus deionizing it. At the end of the cycle, the electrodes are discharged, releasing the adsorbed ions into the brine flow as a by-product. The origin of salt adsorption is not trivial, and resides in the process of charge storage into the electric double layers (EDL) which form at the interface between the carbon and the solution [11]. The charge in the diffuse part of the EDL comes both from an
PACS numbers: 82.45.-h, 73.30.+y, 82.60.Lf, 88.05.De

increase of the concentration of counterions and a decrease of the concentration of coions, but the latter contribution becomes smaller than the former for increasing voltage, resulting in a net ion concentration increase (see Fig. 1), i.e., a salt storage into the EDLs in the couple of electrodes. Other accumulator technologies, besides supercapacitor technology, have been used with the CDI principle, in

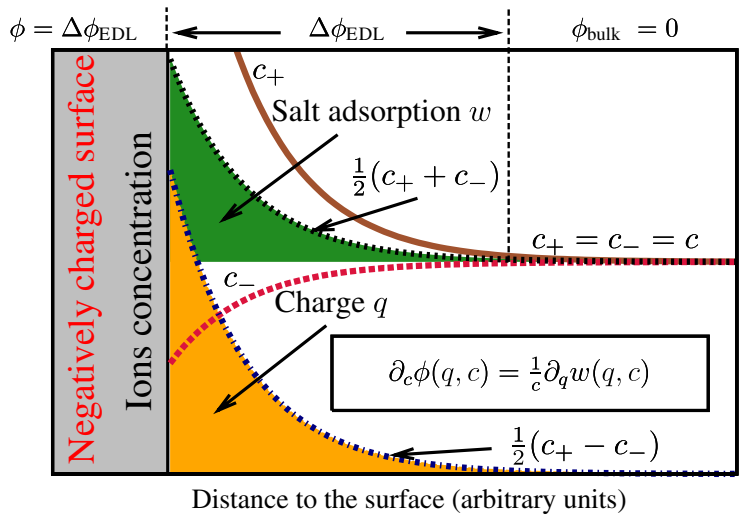

FIG. 1 (color online). Schematic representation of the (dimensionless) quantities of the EDL that are connected by Eq. (4), which is shown in a simplified version (neglecting steric effects, i.e., assuming $\nu=0$; see main text). The specific (per unit surface) amounts of charge $(q)$ and salt $(w)$ adsorbed into the EDL are proportional to the integrals over the whole space of $1 / 2\left(c_{+}-c_{-}\right)$and $1 / 2\left(c_{+}+c_{-}\right)$, respectively. $\left(c_{+}\right.$and $c_{-}$are the concentrations of counterions and coions for a negatively charged surface, respectively. Far from the surface, they are equal to the bulk concentration $c$.) The voltage difference between the solid surface $\phi$ and the bulk $\phi_{\text {bulk }}$ is equal to the voltage drop in the EDL, $\Delta \phi_{\mathrm{EDL}}$. 
particular, battery electrodes [12] and membrane-based ion-selective electrodes [13].

The recently proposed "capacitive mixing" (or capmix) technique [14-18] is aimed at the energy extraction from salinity differences. Similarly to accumulator-based deionization, a capmix process starts charging the electrodes while dipped in salt water. After this phase, the solution in the cell is changed by fresh water in an open circuit, thus keeping constant the stored charge in the electrodes. Because of the concentration change, the cell voltage rises, therefore increasing the stored energy. The surplus energy is then extracted by discharging the cell. The electrodes that have been used for capmix include chemically inert porous materials [14,15,19], membrane-based ionselective electrodes [17], and battery electrodes [20]. In order to include the last technique, the name "accumulator mixing" would be more appropriate.

Although CDI and capmix appear to be the reverse of each other, at least at the operative level, the cycles that have been investigated up to now are not reversible, as two solutions with different salinities always get into contact and irreversibly mix, at least partially, during the solution exchanges [14,21]. However, Boon and van Roij [16] have recently proposed a different cycle that is in principle reversible, although it is not clear whether this new strategy can be easily implemented experimentally. An important difference between CDI and capmix is that they are based on two different physical properties, namely, the salt adsorption during the charge and the dependence of the cell voltage on the salt concentration, respectively.

In this Letter, we derive a relation between these two properties, showing that CDI and capmix are fundamentally connected (see Fig. 1). In particular, our result allows translation of technical improvements of CDI into improvements in capmix; e.g., a material that works well for CDI will also work well for capmix, and vice versa. Moreover, it can be used to make a quantitative prediction, based on CDI performances, of capmix performances, and vice versa. We also check the relation with experimental data, already reported in the literature, showing excellent agreement. Finally, we show that the relation is verified by some paradigmatic physical models describing various kinds of electrodes, including ideally polarizable and nonpolarizable, and for ion-selective electrodes. This makes the analyzed relation a very useful tool for the study of EDLs and for the development of new models [11], a fundamental concept in any field of interface science.

We consider a generic cell composed of two electrodes, with a specific (per unit mass or surface of electrode) charge $Q$, and a bulk solution with mole fraction of ions $x=N / M, N$ and $M$ being the number of moles of salt and solvent, respectively. The expression relating the potential between the two electrodes of the generic cell $V_{\text {cell }}(Q, x)$ and the specific amount of adsorbed salt $W(Q, x)$ is obtained by calculating the (mechanical and electrical) energy consumed in the four-step cycle described in the Supplemental Material [22]. Briefly, it includes the processes of electrically charging and discharging the electrodes, and the equilibration of the salt concentration in the cell with the reservoir, through a semipermeable membrane. The membrane allows the solvent to pass, while it stops salt ions. The osmotic flow is considered to be a mechanical work, while the charge and discharge phases are considered to be an exchange of electrical energy. The cycle is performed at constant temperature, and is reversible [21], given that all the phases are performed slowly enough. Therefore, the (mechanical and electrical) energy lost during the cycle is equal to zero.

The analysis of this cycle leads to the following relation (see Supplemental Material [22]), which constitutes the subject of this Letter, connecting the potential to the salt adsorption,

$$
\partial_{x} V_{\text {cell }}(Q, x)=\partial_{x} \Pi(x) \frac{\partial_{Q} W(Q, x)}{x},
$$

where $\Pi(x)$ is the osmotic pressure of ions in the bulk solution, expressed in $\mathrm{J} / \mathrm{mol}$. It is important to point out that, as usual, partial derivatives are made keeping constant the other independent variable. For example, the derivative of the cell potential on the left-hand side of Eq. (1) is made at constant stored charge, i.e., in open circuit. On the right-hand side, the change of adsorbed salt with charge is evaluated at constant bulk salt concentration.

Equation (1) establishes a relation between the dependence of the cell voltage on the electrolyte concentration of the bulk solution at constant charge $\left[\partial_{x} V_{\text {cell }}(Q, x)\right]$, and the variation of the number of ions (both coions or counterions) stored in the electrodes, with the stored charge, at a given electrolyte concentration $\left[\partial_{Q} W(Q, x)\right]$. We stress that no assumption has been made on the nature of the electrodes nor the kind of charge storage mechanism, and only thermodynamic arguments were used for its derivation.

In the following, we assume that the solute dissociates into two monovalent ions. An approximation can be obtained assuming that the solution is ideal $[\Pi(x)=$ $2 R T x][23]$,

$$
\partial_{C} V_{\text {cell }}(Q, C)=2 R T \frac{\partial_{Q} W(Q, C)}{C},
$$

where $C$ is the concentration (in $\mathrm{mM}$ ), $R$ is the gas constant, and $T$ is the absolute temperature.

The voltage rise in a capmix experiment can be obtained by integrating Eq. (2) with respect to $C$, from $C_{A}$ to $C_{B}$, representing, respectively, the concentrations of the fresh and salt water, e.g. $C_{A}=20 \mathrm{mM}$ and $C_{B}=500 \mathrm{mM}$. It is convenient to introduce the differential charge efficiency $\lambda(Q, C)=F \partial_{Q} W(Q, C) \quad(F$ is the Faraday constant $)$, which represents the change in the number of moles of adsorbed salt per mole of charges stored in the electrodes [24]. In most practical cases, the variation of $\lambda(Q, C)$ in the 
range $\left(C_{A}, C_{B}\right)$ is negligible with respect to the variation of $1 / C$, and thus we can assume that $\lambda(Q, C)$ is constant with respect to the integration variable $C$, i.e. $\lambda\left(Q, C_{A}\right) \simeq$ $\lambda(Q, C) \simeq \lambda\left(Q, C_{B}\right)$ for any $C$ between $C_{A}$ and $C_{B}$. The approximate result of the integration is

$$
\Delta V_{\text {cell }}\left(Q, C_{A}, C_{B}\right) \simeq 2 \frac{R T}{F} \ln \left(\frac{C_{B}}{C_{A}}\right) \lambda\left(Q, C_{A}\right),
$$

where $\Delta V_{\text {cell }}\left(Q, C_{A}, C_{B}\right)=V_{\text {cell }}\left(Q, C_{A}\right)-V_{\text {cell }}\left(Q, C_{B}\right)$ is the voltage rise upon a concentration decrease. In Eq. (3), we identify a factor that represents the variation of the potential of an electrochemical cell, when the concentration of the solution is changed from $C_{B}$ to $C_{A}$, as obtained by Nernst equation. The actual voltage rise is obtained by multiplying the Nernst potential by the efficiency $\lambda(Q, C)$, which has already been described as the efficiency of salt adsorption. Thus, this equation defines a relation between the voltage rise and the differential charge efficiency, which are the physical parameters of interest for capmix and CDI, respectively.

We have verified Eq. (3) with experimental data, already reported in the literature [25], concerning a CDI experiment with activated carbon electrodes. In that case, the salt adsorption was measured for sodium chloride solutions at $C_{A}=5 \mathrm{mM}$ and $C_{B}=20 \mathrm{mM}$. We evaluated the differential charge efficiency (different from the charge efficiency defined in [25]) by first smoothing the experimental data (see Supplemental Material [22]). In the same experiment, the voltage versus charge curve was also measured at $C_{A}=5 \mathrm{mM}$ and $C_{B}=20 \mathrm{mM}$, and from these data we inferred the voltage rise upon concentration change $\Delta V_{\text {cell }}\left(Q, C_{A}, C_{B}\right)$. Figure 2 shows the comparison of the two sides of Eq. (3) measured in the experiment, showing very good agreement between the two routes. The fact that the two data sets are obtained from different types of measurements $\left(V_{\text {cell }}, Q\right.$, and $W$ were measured indepen-

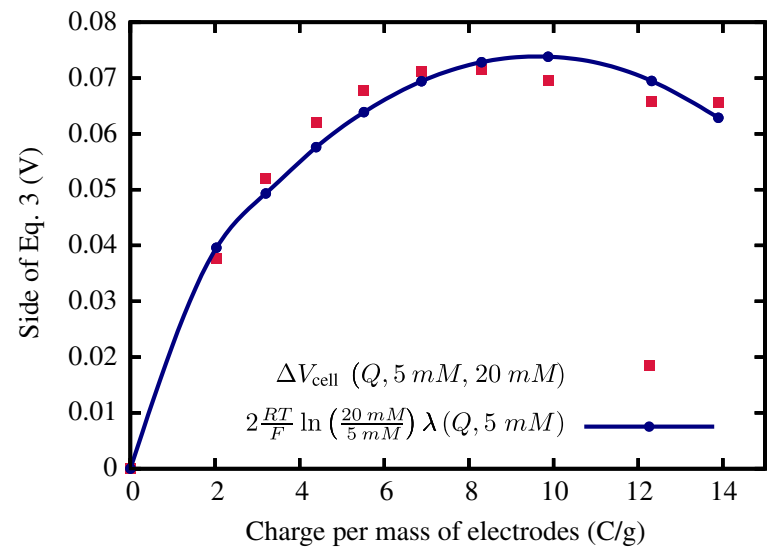

FIG. 2 (color online). Experimental verification of Eq. (3), in the case of a cell with two activated carbon electrodes in a sodium chloride solution, with concentrations $20 \mathrm{mM}$ and $5 \mathrm{mM}$. The data shown are the two sides of Eq. (3), calculated from the experimental data reported in Ref. [25]. dently) makes this comparison an excellent verification of Eq. (3).

For its application to the study of the properties and modeling of EDLs on polarizable electrodes [11,16,26,27], it is convenient to rewrite Eq. (1) in such a way that it describes a single electrode, introducing some dimensionless quantities,

$$
\partial_{c} \phi(q, c)=\frac{\partial_{q} w(q, c)}{(1-\nu) c},
$$

where $\phi(q, c)=V / V_{\mathrm{T}}, V_{\mathrm{T}}=R T / F, c=C / \tilde{C}$, with $\tilde{C}$ an arbitrary concentration reference. $q=Q / 2 F \tilde{\lambda}_{D} \tilde{C}$ and $w=$ $W / 2 \tilde{\lambda}_{D} \tilde{C}$ are dimensionless charge and ion adsorption densities in the diffuse part of the EDL, respectively. $\tilde{\lambda}_{D}=1 / \sqrt{8 \pi \lambda_{B} N_{A} \tilde{C}}$ is the Debye length, $N_{A}$ is the Avogadro number, and $\lambda_{B}$ is the Bjerrum length, which, at room temperature in water is $\lambda_{B} \simeq 0.72 \mathrm{~nm}$. $\nu=$ $2 c / c_{\max }$ is a packing parameter due to steric effects of ions [26,27], which defines a maximum concentration $c_{\max }$.

Steric effects in the EDL can be accounted for through the widely used Bikerman lattice model [26-29], which provides the following expressions for the voltage-charge relation, and ions adsorption in the diffuse part of the EDL:

$$
\begin{gathered}
\Delta \phi_{D}(q, c)=-2 \operatorname{sgn}(q) \sinh ^{-1} \sqrt{\frac{\exp \left(\frac{q^{2} \nu}{2 c}\right)-1}{2 \nu}}, \\
w(q, c)=-2 \sqrt{c} \operatorname{sgn}(q) \int_{0}^{\Delta \phi_{D}(q, c)} \frac{\sinh ^{2} \frac{\varphi}{2}}{1+2 \nu \sinh ^{2} \frac{\varphi}{2}} \\
\times \frac{(1-\nu) d \varphi}{\sqrt{\frac{2}{\nu} \ln \left(1+2 \nu \sinh ^{2} \frac{\varphi}{2}\right)}} .
\end{gathered}
$$

Here, $\Delta \phi_{D}(q, c)$ is the dimensionless voltage drop across the diffuse part of the EDL. The total voltage drop in the $\mathrm{EDL}$ is $\phi(q, c)=\Delta \phi_{\mathrm{EDL}}(q, c)=\Delta \phi_{D}(q, c)+$ $\Delta \phi_{\mathrm{St}}(q, c)$, where $\Delta \phi_{\mathrm{St}}(q, c)=-\frac{\lambda_{\mathrm{St}}}{\hat{\lambda}_{D}} q$ and $\lambda_{\mathrm{St}}$ are the voltage drop and effective length of the Stern layer, respectively. It can be shown that Eqs. (5) and (6) verify Eq. (4). Furthermore, in the limit $\nu \rightarrow 0$, when steric effects are neglected, Eqs. (5) and (6) reduce to the classical Gouy-Chapman-Stern (GCS) model, defined by [11,30]

$$
\begin{gathered}
\Delta \phi_{D}(q, c)=-2 \sinh ^{-1} \frac{q}{2 \sqrt{c}}, \\
w(q, c)=\sqrt{4 c+q^{2}}-2 \sqrt{c},
\end{gathered}
$$

which verify Eq. (4) in the same limit. Both the GCS and the Bikerman models assume thin double layers. In the opposite situation, we can use the so-called modified Donnan model [31], valid for porous materials in which the Debye length much exceeds the characteristic pore size. The details for 
this case, which also verifies Eq. (4), are reported in the Supplemental Material [22].

CDI and capmix have also been performed with assemblies composed of an ion-selective membrane and an activated carbon electrode [13,17]. When considered as a whole, also this kind of complex electrode satisfies the relation expressed by Eq. (3). In particular, the potential difference established between two reservoirs with different concentrations $C_{A}$ and $C_{B}$ separated by a nonideal ionselective membrane is usually written as [32]

$$
\Delta V=\frac{2 R T}{F} \ln \left(\frac{C_{B}}{C_{A}}\right)\left(t_{+}-t_{-}\right),
$$

where $t_{+}$and $t_{-}$are the transport numbers of cations and anions inside the membrane, respectively. A membrane is called ideal when one of the transport numbers is 1 , while the other vanishes. In this situation, Eq. (9) reduces to the Nernst equation. Direct comparison of Eqs. (9) and (3) leads us to pose the following identity:

$$
\lambda(Q, C) \equiv\left(t_{+}-t_{-}\right)
$$

This identity relates the concepts of EDL and ion-selective membrane, in the sense that inside an EDL the transport numbers of anions and cations are different from their bulk values because of their modified concentrations. Therefore, this comparison illustrates the evolution of the charge efficiency with the charge inside the EDL: when the EDL is only slightly charged, $\lambda(Q, C)$ is also small, similarly to a membrane with a small number of fixed charges and hence a low selectivity, quantified by the difference in transport numbers. In this situation, both types of ions can pass freely through the interface and there is no net transport of salt. On the contrary, when an EDL or a membrane is highly charged, the difference in transport numbers and the charge efficiency take values close to 1 , and the current is carried by only one type of ion, leading to the imbalance between counterion adsorption and coion repulsion responsible for the storage of neutral salt in the EDL or in one side of a membrane. Such a relation between the concepts of EDL and ion-selective membranes was previously proposed by Ohshima and Ohki [33].

When battery electrodes are used for CDI [12] or capmix [20], the electrodes store ions by means of a redox reaction. The voltage dependence on the concentration is described by the Nernst equation,

$$
V(Q, C)=V_{0}-2 \frac{R T}{F} \ln \left(\frac{C}{\tilde{C}}\right)
$$

The amount of adsorbed salt is evaluated by observing that a couple of positive and negative ions react and are adsorbed for each electron that is moved from the positive to the negative electrode,

$$
W(Q, C)=W_{0}+\frac{Q}{F}
$$

Together, these two expressions satisfy Eq. (2).

Summarizing, we have analyzed a relation connecting two different concepts: the dependence of the voltage on bulk salt concentration and the salt adsorption upon charging an electrochemical cell. Capacitive mixing relies on the first dependence: in particular, the power output is roughly proportional to the square of the voltage rise. CDI relies on the second quantity, and the charge efficiency represents the efficiency of desalination of a given device. Our relation demonstrates that an electrode setup having a good performance for CDI should also work well for capmix, and vice versa. Therefore, experimental and theoretical findings obtained for the salt adsorption immediately lead to conclusions concerning the dependence of the potential on concentration, and vice versa. This holds also for complex electrode setups, and this enables the maximization of the efficiency for both capmix and CDI-like processes. Moreover, this relation is of interest in the development and study of general EDL models.

We thank Marek Bryjak and René van Roij for useful discussions. Financial support from the European Union Seventh Framework Program (FP7/2007-2013) under Agreement No. 256868 is gratefully acknowledged. R. A. R. acknowledges support from Regione Lombardia (Accordo per lo Sviluppo del Capitale Umano nel Sistema Universitario Lombardo). F. M. and D. S. acknowledge support of Cariplo Foundation Materiali Avanzati-2011, Project No. 2011-0336.

[1] S. A. Kalogirou, Prog. Energy Combust. Sci. 31, 242 (2005).

[2] T.-S. Chung, X. Li, R. C. Ong, Q. Ge, H. Wang, and G. Han, Current Opinion in Chemical Engineering 1, 246 (2012).

[3] B. Logan and M. Elimelech, Nature (London) 488, 313 (2012).

[4] R. E. Pattle, Nature (London) 174, 660 (1954).

[5] O. Levenspiel and N. de Vevers, Science 183, 157 (1974).

[6] S. Loeb, Science 189, 654 (1975).

[7] J.W. Post, H. V.M. Hamelers, and C.J.N. Buisman, Environ. Sci. Technol. 42, 5785 (2008).

[8] J. N. Weinstein and F. B. Leitz, Science 191, 557 (1976).

[9] M. Olsson, G. L. Wick, and J. D. Isaacs, Science 206, 452 (1979).

[10] Y. Oren, Desalination 228, 10 (2008).

[11] M. Z. Bazant, K. Thornton, and A. Ajdari, Phys. Rev. E 70, 021506 (2004).

[12] M. Pasta, C.D. Wessells, Y. Cui, and La Mantia, Nano Lett. 12, 839 (2012).

[13] P. M. Biesheuvel and A. van der Wal, J. Membr. Sci. 346, 256 (2010).

[14] D. Brogioli, Phys. Rev. Lett. 103, 058501 (2009). 
[15] D. Brogioli, R. Zhao, and P.M. Biesheuvel, Energy Environ. Sci. 4, 772 (2011).

[16] N. Boon and R. van Roij, Mol. Phys. 109, 1229 (2011).

[17] B. B. Sales, M. Saakes, J. Post, C. J. N. Buisman, P. M. Biesheuvel, and H. V. M. Hamelers, Environ. Sci. Technol. 44, 5661 (2010).

[18] M. F. M. Bijmans, O. S. Burheim, M. Bryjak, A. Delgado, P. Hack, F. Mantegazza, S. Tennisson, and H. V. M. Hamelers, Energy Procedia 20, 108 (2012).

[19] R. A. Rica, D. Brogioli, R. Ziano, D. Salerno, and F. Mantegazza, J. Phys. Chem. C 116, 16934 (2012).

[20] F. La Mantia, M. Pasta, H. D. Deshazer, B. E. Logan, and Y. Cui, Nano Lett. 11, 1810 (2011).

[21] P. M. Biesheuvel, J. Colloid Interface Sci. 332, 258 (2009).

[22] See Supplemental Material at http://link.aps.org/ supplemental/10.1103/PhysRevLett.109.156103 for details on the derivation of Eq. (1), the experimental verification of Eq. (3), and the details of the modified Donnan model for strongly overlapping EDLs.
[23] L.E. Reichl, A Modern Course in Statistical Physics (E. Arnold, London, 1987).

[24] P. M. Biesheuvel, B. van Limpt, and A. van der Wal, J. Phys. Chem. C 113, 5636 (2009).

[25] R. Zhao, P. M. Biesheuvel, H. Miedema, H. Bruning, and A. van der Wal, J. Phys. Chem. Lett. 1, 205 (2010).

[26] M.Z. Bazant, M.S. Kilic, B.D. Storey, and A. Ajdari, Adv. Colloid Interface Sci. 152, 48 (2009).

[27] H. Wang and L. Pilon, J. Phys. Chem. C 115, 16711 (2011).

[28] J. J. Bikerman, Philos. Mag. 33, 384 (1942).

[29] M. S. Kilic, M. Z. Bazant, and A. Ajdari, Phys. Rev. E 75, 021503 (2007).

[30] P. M. Biesheuvel, Y. Fu, and M.Z. Bazant, Phys. Rev. E 83, 061507 (2011).

[31] P. M. Biesheuvel, R. Zhao, S. Porada, and A. van der Wal, J. Colloid Interface Sci. 360, 239 (2011).

[32] N. Lakshminarayanaiah, Chem. Rev. 65, 491 (1965).

[33] H. Ohshima and S. Ohki, Biophys. J. 47, 673 (1985). 